\title{
XANES study of oxygen doping in 1-2-3 system
}

\author{
K V R RAO and K B GARG \\ Department of Physics, University of Rajasthan, Jaipur 302004, India
}

Abstract. Studies on $\mathrm{X}$-ray absorption near-edge structure (XANES) using full multiple scattering approach on $\mathrm{YBa}_{2} \mathrm{Cu}_{3} \mathrm{O}_{7-x}$ with $x=0,0 \cdot 5$ and $1, \mathrm{CuO}$ and $\mathrm{Cu}_{2} \mathrm{O}$ are reported. The MS-XANES calculations confirm that removal/addition of oxygen between $x=0.0$ and 1.0 appears to be confined to $\mathrm{Cu}$ chain sites only.

Keywords. XANES; oxygen doping.

\section{Introduction}

Since the discovery of a new class of oxide superconductors, study of their ground-state electronic configuration is considered crucial in determining the pairing mechanism responsible for superconductivity. It is now well established that $\mathrm{YBa}_{2} \mathrm{Cu}_{3} \mathrm{O}_{7}(\mathrm{YBCO} 7)$ is an orthorhombic phase where the unit cell can be regarded as a distorted perovskite structure with ordered vacancies with $\mathrm{Cu}(2)$ site forming a square pyramidal arrangement (planes) and $\mathrm{Cu}(1)$ site having a square planar coordination (chains). $\mathrm{A}$ transition in orthorhombic-tetragonal occurs at approximately $\mathrm{YBa}_{2} \mathrm{Cu}_{3} \mathrm{O}_{6.5}$ (YBCO6.5) with progressive loss of chains alternately and complete removal of oxygen from chains forms a tetragonal $\mathrm{YBa}_{2} \mathrm{Cu}_{3} \mathrm{O}_{6}$ (YBCO6). The chemical environment around $\mathrm{Cu}(\mathrm{I})$ site is thus modified when the oxygen content is decreased.

Experimental investigations on high temperature superconductors with variable oxygen content by $\mathrm{CuK}$-edge X-ray absorption near edge structure (XANES) have been performed to determine the local electronic structure of $\mathrm{Cu}$ ions and local atomic distribution. This method enables the study of local structure around the absorbing atom and the changes in spectra with stoichiometry variation especially in superconductors with variable oxygen content.

However, the main controversy concerning peak $D$ appearing at about $5 \mathrm{eV}$ beyond the main peak led to many interpretations. Alp et al $(1987,1988)$ and Oyanagi et al (1987) ascribed this to $\mathrm{Cu}^{3+}$ state. However, experiments by Bianconi et al (1987a-c) using XPS and $\mathrm{Cu} \mathrm{L}_{111}$ XANES techniques established that there is no trivalent state of copper and that it arises due to holes on the oxygen valence band and its coupling with $\mathrm{Cu} 3 d$ electrons giving rise to superconductivity in these systems. Subsequently Garg et al (1988b) calculated MS-XANES for YBCO7 and reported quantitative agreement between experimental and calculated spectra. These conclusions received support from a number of spectroscopic investigations (Baudlet et al 1987; Fujimori et al 1987; Horn et al 1988; Saachi et al 1988; Takahashi et al 1988; Tranquada et al 1988; Heald et al 1988; Nucker et al 1989). No quantitative data analysis of CuK-edge XANES for YBCO6.5 and YBCO6 has however been reported. XANES analysis has been performed to establish that oxygen is absent only from chains and that the monovalent nature of copper in these perovskites is largely confined to the $\mathrm{Cu}(1)$ sites.

In this paper, we report our data analysis of the CuK-edge XANES spectra of YBCO7, YBCO6.5 and YBCO6 using single-electron MS-XANES calculation. The XANES spectra are interpreted in terms of multiple scattering of the photoelectron 
within a cluster of finite size. We have considered the local structure due to the orthorhombic or tetragonal, space group crystalline structure and the presence of two non-equivalent sites $\mathrm{Cu}(1)$ and $\mathrm{Cu}(2)$ of the $\mathrm{Cu}$ ions in the unit cell. The calculated spectra are compared with experiments and the various features are associated with valence states of copper or MS resonances. The electron approximation used for the present XANES calculations assumes a single configuration of passive valence electrons.

\section{Experimental}

YBCO compounds were prepared by the usual solid-state reaction. Stoichiometric proportions of high purity $\mathrm{Y}_{2} \mathrm{O}_{3}, \mathrm{BaCo}_{3}$ and $\mathrm{CuO}$ were taken, dried at $100^{\circ} \mathrm{C}$ overnight, calcined at $950^{\circ} \mathrm{C}$ for $15 \mathrm{~h}$ and annealed in oxygen at $450^{\circ} \mathrm{C}$ for $14 \mathrm{~h}$. The oxygen content ' $x$ ' was varied by controlling the cooling conditions. The sample $6.95 \pm 0.05$ was prepared accordingly. The oxygen-deficient insulating sample $(\mathrm{O}=6.5 \pm 0 \cdot 1)$ was prepared by similar annealing, heating up to $700^{\circ} \mathrm{C}$ for $5 \mathrm{~h}$ and subsequent quenching from $700^{\circ} \mathrm{C}$ to liquid nitrogen temperature. The sample of oxygen content $\mathrm{O}=6.0 \pm 0.1$ was prepared by annealing under nitrogen flow for $30 \mathrm{~h}$. The oxygen content was determined by using iodometric technique and the titration repeated several times to confirm oxygen content. It was found that oxygen content values are reliable up to \pm 0.1 for YBCO6.5 and YBCO6 and to \pm 0.05 for the YBCO7 sample. Resistivity was measured with a standard four-probe method and the sample with $\mathrm{O}=6.95 \pm 0.05$ showed a transition temperature $T_{c}$ (zero resistance) around $89 \mathrm{~K}$. No superconductivity was observed for samples with oxygen content $6 \cdot 5 \pm 0 \cdot 1$ and $6 \cdot 0 \pm 0 \cdot 1$ down to $50 \mathrm{~K}$. Phase purity was confirmed by X-ray diffraction analysis.

The CuK-XANES measurements were made using a laboratory EXAFS system with a resolution of $0.8 \mathrm{eV}$ in this energy range. The spectrum was measured several times in each case to achieve an accuracy better than $0.2 \%$ at the main peak of CuK-edge.

\section{Results and discussion}

Our measured spectra for the YBCO systems are shown in figure 1 along with $\mathrm{CuO}$ and $\mathrm{Cu}_{2} \mathrm{O}$. These results agree with those reported earlier (Bianconi et al 1987a-c; Garg et al 1988a-c; Saachi et al 1988; Horn et al 1988; Kosugi et al 1989). Peak $A^{\prime}$ appearing prominently in $\mathrm{Cu}_{2} \mathrm{O}$ is characteristic of linearly coordinated compounds. It is also seen in the spectra of YBCO6.5 and YBCO6. The shoulder $A$, following Bair and Goddard (1980) can be said to be a $1 s-4 p$ ligand-to-metal shake-down satellite. Kosugi et al (1989) ascribed shoulder $B^{\prime}$ to $1 s-4 p$ chain-like transition in the chains and is not understood very well. Peak $B$ in $\mathrm{YBCO} 7$ is also attributed to $1 s-4 p$ transition. The main peak $C$ is well known to possess $1 s-4 p$ dipole transition that appears in all $\mathrm{Cu}$ compounds. Peak $D$ which has been cited as the presence of $\mathrm{Cu}^{3+}$ in the superconducting YBCO7 system earlier (Alp et al 1987, 1988; Oyanagi et al 1987, 1988 ) is present in the spectra of YBCO6.5 and insulating YBCO6 as well and implies that peak $D$ cannot signify the presence of $\mathrm{Cu}$ in the trivalent state. 


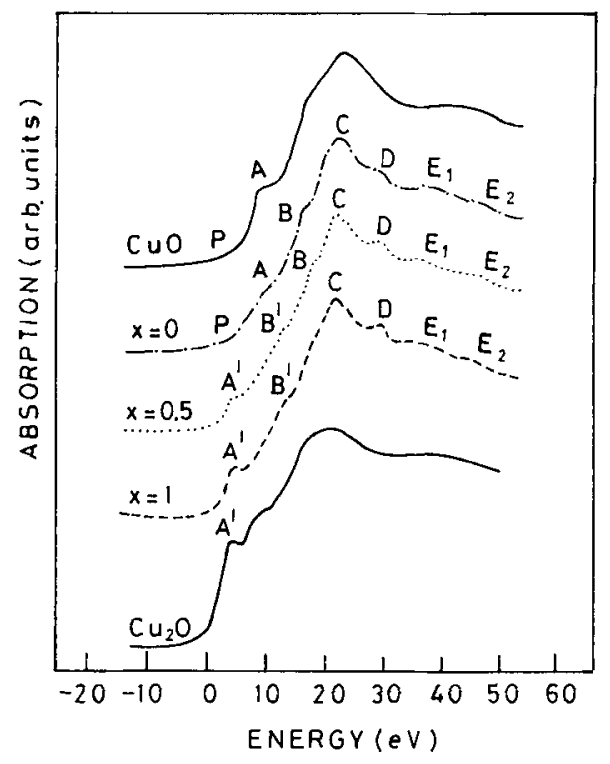

Figure 1. Measured CuK-edge XANES spectra of $\mathrm{YBa}_{2} \mathrm{Cu}_{3} \mathrm{O}_{7-x}(x=0,0 \cdot 5,1)$ together with $\mathrm{CuO}$ and $\mathrm{Cu}_{2} \mathrm{O}$.

From experimental results, one can draw certain conclusions. Peak $A^{\prime}$ which indicates presence of monovalent $\mathrm{Cu}$ grows in intensity for YBCO6.5 and YBCO6 systems. This reveals that $\mathrm{Cu}^{1+}$ content in $\mathrm{YBCO}$ system keeps on increasing as the oxygen content decreases. On the other hand, the peak/shoulder $A$ which confirms the presence of $\mathrm{Cu}^{2+}$ appears in case of YBCO7. This evidently means that decrease of oxygen leads to conversion of $\mathrm{Cu}^{2+}$ into $\mathrm{Cu}^{1+}$ progressively. In other words, the octahedral oxygen environment as in the case of $\mathrm{CuO}$ progressively converts into two-fold linear coordination as in $\mathrm{Cu}_{2} \mathrm{O}$. It is then natural to ask which copper site is losing its oxygen neighbours and in what way. Earlier reports (Saachi et al 1988; Horn et al 1988; Tranquada et al 1988; Kosugi et al 1989) state that oxygen starts disappearing first from the alternate chains around $\mathrm{Cu}(1)$ site in $\mathrm{YBCO} 6.5$ and finally all the chains disappear in YBCO6.

We have carried out multiple scattering calculations for all the measured systems. Non-self consistent XANES calculations have been carried out using the MS-XANES programme of Durham et al (1982). The interpretation of CuK-edge XANES in YBCO compounds is complex because of large number of vacancies, the orthorhombic-tetragonal distortion and the presence of two non-equivalent $\mathrm{Cu}$ sites in the unit cell.

For the $\mathrm{YBCO} 65$ system, oxygen was removed from the alternate $\mathrm{Cu}-\mathrm{O}$ chains around $\mathrm{Cu}(1)$ site. This evidently resulted in the unit cell becoming double the size $\mathrm{Y}_{2} \mathrm{Ba}_{4} \mathrm{Cu}_{6} \mathrm{O}_{13}$ as shown in figure 2. For $\mathrm{YBCO6}$, oxygen was removed from the remaining chains as well and the unit cell became $\mathrm{YBa}_{2} \mathrm{Cu}_{3} \mathrm{O}_{6}$. In all the three cases of $\mathrm{YBCO}$ calculated above there were two non-equivalent $\mathrm{Cu}$ sites. In $\mathrm{YBCO}$, $\mathrm{Cu}(1)$ has a four-fold square planar coordination while $\mathrm{Cu}(2)$ site forms a square pyramidal configuration. In $\mathrm{YBCO}$, the $\mathrm{Cu}(2)$ coordination remains in tact and square planar coordination for $\mathrm{Cu}(1)$ degenerates into a simple linear coordination. In YBCO6.5, 


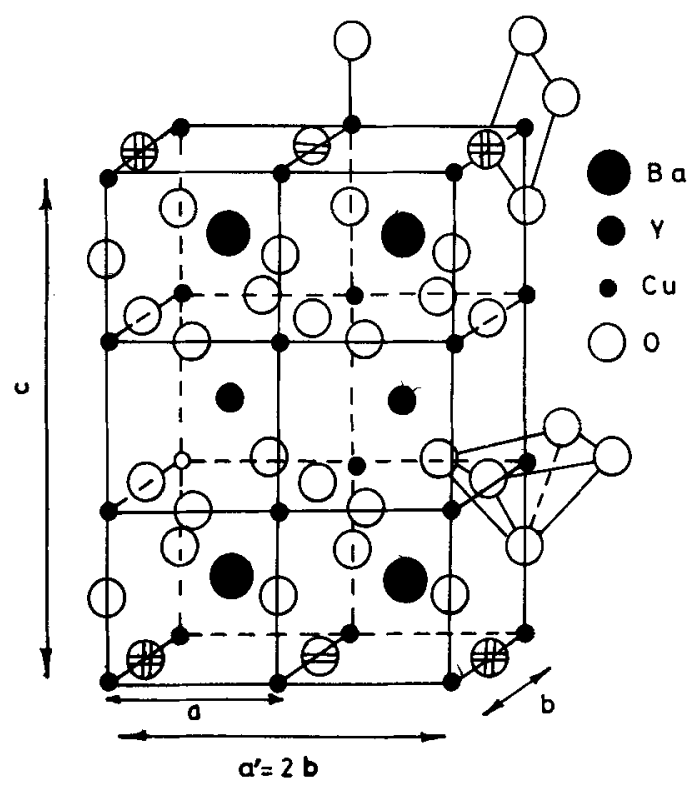

Figure 2. Crystal structure with oxygen $(\theta)$ disappearing from alternate chains in $\mathrm{YBa}_{2} \mathrm{Cu}_{3} \mathrm{O}_{6 \cdot 5}$ and complete disappearance of oxygen (1) from alternate chains in $\mathrm{YBa}_{2} \mathrm{Cu}_{3} \mathrm{O}_{6}$.

there are two types of coordination at $\mathrm{Cu}(1)$ site, four-fold square planar as in $\mathrm{YBCO} 7$ and two-fold linear as in YBCO6. For actual calculations, the clusters for YBCO7 were built using unit cell coordinates of Capponi et al (1987) in which the puckering of planes was also considered. Clusters for YBCO6.5 and YBCO6 cases were also deduced from the same. The clusters for calculation for $\mathrm{CuO}$ and $\mathrm{Cu}_{2} \mathrm{O}$ were built from the coordinates given in the Wycoff Crystal Structures.

Our calculated spectra (dotted) are shown in figure 3 . The calculations were made only up to an energy range of about $40 \mathrm{eV}$. Our calculated spectra for $\mathrm{CuO}$ and $\mathrm{Cu}_{2} \mathrm{O}$ are almost identical to those reported by Norman et al (1985). Similarly our calculated spectra for YBCO7 are also nearly identical to that reported by Garg et al (1988).

The calculations were carried out for each non-equivalent $\mathrm{Cu}$ sites and the spectra shown in figure 3 are the weighted average of these in the ratio of $1: 2$ for $\mathrm{Cu}(1)$ and $\mathrm{Cu}(2)$ sites. Peak $A$ which is signature of $\mathrm{Cu}^{2+}$ as in $\mathrm{CuO}$ is clearly present in the YBCO 7 spectrum but fades out in the YBCO6.5 and YBCO6 spectra. Peak $A^{\prime}$, on the other hand, which constitutes $\mathrm{Cu}^{1+}$ as in $\mathrm{Cu}_{2} \mathrm{O}$ is absent in the YBCO7 spectrum but appears in YBCO6.5 and YBCO6 and has tremendous intensity in the YBCO6 system. This confirms the conjecture that $\mathrm{Cu}^{2+}$ progressively changes to $\mathrm{Cu}^{1+}$ as oxygen doping goes down. Also, the calculations have been made for YBCO6.5 and YBCO6 by removing only oxygen from the chain sites. These results indicate that chains lose oxygen as the oxygen content in YBCO is progressively decreased.

The continuous spectra in figure 3 represent the measured spectra in each case which agree fairly well with the calculated spectra. In addition changes in intensities of the peaks $A$ and $A^{\prime}$ represent the signature of $\mathrm{Cu}^{2+}$ and $\mathrm{Cu}^{1+}$, respectively. There is also an interesting behaviour of fine structure $B^{\prime}$ which is said to arise from $1 s-4 p$ 


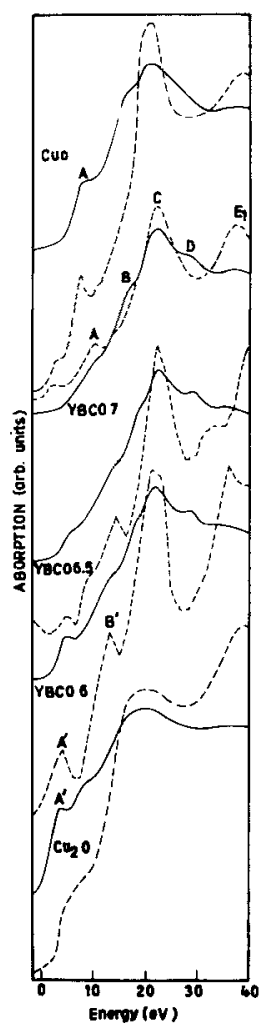

Figure 3. Calculated (-...-) and measured (-) for K-edge XANES spectra of $\mathrm{YBa}_{2} \mathrm{Cu}_{3} \mathrm{O}_{7-x}(x=0,0 \cdot 5,1)$ together with $\mathrm{CuO}$ and $\mathrm{Cu}_{2} \mathrm{O}$.

transition in the chain sites. Its appearance in the oxygen-deficient samples indicates the presence of $\mathrm{Cu}^{1+}$ in both experimental and calculated spectra. Its absence in YBCO7 further lends support to this conjecture.

There are, however, certain weaknesses of the one-electron non-self-consistent potential MS calculations. In the one-electron approximation a single-electron configuration of the passive valence electrons is considered. This is a fully relaxed configuration in the presence of the core hole according to the XPS data shown (Bianconi et al 1987a). We have used the Cu3 $d^{10} 4 s^{1}$ of passive valence electrons.

Since XANES calculations do not include the energy-dependent exchange interaction between excited electrons and valence electrons, it is necessary to expand the energy scale of calculated spectrum by an empirical factor. In the present case, the expansion of energy scales for YBCO7, YBCO6.5 and YBCO6 comes out to be a factor of $1 \cdot 15$, in each case to compare these with the calculated spectrum.

All the spectral features of the experiment agree with the calculated spectra but the agreement is not clear in the case of relative intensities and energy positions. An enhancement of peak $A$ in both experimental and calculated spectra of YBCO6.5 in intensity is expected since the excitation energy of the ligand electron and intensity increases due to greater overlap between the ligand orbital and the $\mathrm{Cu}$ core states as the decreasing oxygen content reduces the binding energy of the ligand electron. No shake- 
down phenomenon is expected in the $3 d^{10}$ state as seen from its absence in both the YBCO6 spectra.

\section{Conclusions}

We have carried out XANES measurements for YBCO7, YBCO6.5 and YBCO6 besides $\mathrm{CuO}$ and $\mathrm{Cu}_{2} \mathrm{O}$ systems. A comparison of the experimental spectra of different samples shows that the $\mathrm{Cu}^{1+}$ content keeps increasing at the cost of $\mathrm{Cu}^{2+}$ as oxygen doping progressively reduces from YBCO7 to YBCO6. The calculated spectra confirms this and also that oxygen disappears from chain sites. The measured and calculated spectra for all systems show reasonable agreement.

\section{Acknowledgements}

We thank Drs S K Joshi and A V Narlikar for advice. Thanks are also due to the University Grants Commission and the Department of Science and Technology for financial support in the form of fellowship and research project.

\section{References}

Alp E E et al 1987 Phys. Rev. B35 7199

Alp E E, Soderholm L, Shenoy G K, Hinks D G, Veal B W and Montano P A 1988 Physica C150 74

Baudlet F et al 1987 Z. Phys. B69 141

Bianconi A et al 1987a Int. J. Mod. Phys. B1 853

Bianconi A, Congiu Castellano A, De Santis M, Delogu P, Gargano A and Giorgi R 1987b Solid State Commun. 631135

Bianconi A, Congiu Castellano A, De Santis M, Rudolf P, Lagarde P and Flank A M 1987c Solid State Commun. 631009

Capponi J J et al 1987 Europhys. Lett. 31301

Durham P J, Pendry J B and Hodges C H 1982 Comput. Phys. Commun. 25193

Fujimori A, Takayama-Muromachi E, Uchida Y and Okai B 1987 Phys. Rev. B35 8814

Garg K B, Singhal R K, Rao K V R, Chandra U, Chauhan H S, Singh J, Jerath K S and Jain D C 1988a Phys. Status Solidi 147343

Garg K B, Bianconi A, Della Longa S, Clozza A, De Santis M and Marcelli A 1988b Phys. Rev. B38 244

Garg K B, Chauhan H S, Jain D C, Chandra U and Singh J 1988c Physica C153-155 121

Heald S M, Tranquada J M, Moodenbaugh A R and Xu Y 1988 Phys. Rev. B38.761

Hewatt A W, Capponi J J, Chailout C, Marezio M M and Hewatt E A 1987 Solid State Commun. 64301 Horn S, Rielly K, Fisk Z, Kwok R S, Thompson J D, Borges H A, Chang C L and Den Boer M L 1988 Phys. Rev. $\mathbf{B 3 8} 2930$

Kosugi N, Kondon H, Tajima $H$ and Kuroda $H$ (Preprint)

Norman D, Garg K B and Durham P J 1985 Solid State Commun. 56895

Nucker N, Fink J, Fuggle J C, Durham P J and Temmerman W M 1989 Phys. Rev. B37 5158

Oyanagi H et al 1987 Jpn J. Phys. 26 L488

Pendry J B 1974 in Low energy electron diffraction (New York: Academic Press)

Saachi M, Corni F, Antonini G M, Calandra C, Matta Cotta F C and Frahm R 1988 Z. Phys. B72 335

Takahashi T et al 1988 Phys. Rev. B37 9788

Tranquada J M, Heald S M, Moodenbaugh A R and Xu Y 1988 Phys. Rev. B38 8893 